\title{
MIR520C Pre-miRNA
}

National Cancer Institute

\section{Source}

National Cancer Institute. MIR520C Pre-miRNA. NCI Thesaurus. Code C82840.

MIR520C is an oligoribonucleotide that is encoded by the human MIR520C gene and has a role in the regulation of gene expression. 